\section{TOXICITY OF AN FC ENGINEERED ANTI-CD40 ANTIBODY IS ABROGATED BY INTRATUMORAL INJECTION AND RESULTS IN DURABLE ANTI-TUMOR IMMUNITY IN PATIENTS}

${ }^{1}$ David Knorr*, 'Jeffrey Ravetch, ${ }^{2}$ Gabriela D'Andrea, ${ }^{2}$ Linda Vahdat, ${ }^{2}$ Christopher Klebanoff, ${ }^{2}$ Mark Robson. ${ }^{1}$ Rockefeller University, New York, USA; ${ }^{2}$ MSKCC, New York, NY, USA

Background Currently approved immune-based therapeutics primarily block inhibitory $\mathrm{T}$ cell checkpoints. Alternative approaches involve activation of immune pathways by agonism of stimulatory receptors, such as CD40. CD40 provides a central mechanism for the activation dendritic cells and is well established in pre-clinical models. Despite its promise, multiple clinical trials with agonistic anti-CD40 antibodies have reported minimal clinical responses and systemic toxicity. Efficient CD40 agonism requires receptor multimerization which we achieved by engineering the human anti-CD40 antibody CP-870,893 with 5 point-mutations in the Fc domain selectively increasing its binding to human FcyRIIB (herein "2141V11"). The re-engineered antibody demonstrated significantly enhanced anti-tumor activity as compared to its parental IgG2 version using a mouse model carrying human Fcy receptors (FcyR) and human CD40 (hFcyR/hCD40) in place of their mouse homologues. ${ }^{1}$ When given systemically, enhanced in vivo activity was accompanied by thrombocytopenia and transaminitis, similar toxicities seen with current clinical anti-CD40 antibodies, resulting from the expression of CD40 on platelets and Kupffer cell activation. As a prelude to clinical studies of 2141-V11 an optimized a dosing and delivery regimen resulting in minimal toxicity with optimal anti-tumor activity was developed, demonstrating that direct intratumoral injection led to potent local and systemic anti-tumor immunity. ${ }^{2}$

Methods We performed pre-clinical toxicology testing in macaques and found no toxicity up to $100 \mathrm{mg} / \mathrm{kg}$ subcutaneously, in contrast to the toxicity observed in hCD $40 / \mathrm{hFcyR}$ mice. CD40 agonistic antibodies require engagement by their $\mathrm{Fc}$ to FcyRIIB to achieve receptor multimerization and agonistic signaling. This lack of toxicity in macaques resulted from the observation that while 2141-V11 can engage the macaque CD40 molecule it does not engage the macaque inhibitory FcyRIIB, highlighting the importance of matching antibodies with their species specific Fc receptors. Full toxicology results will be reported at the annual meeting. We are now testing intratumoral 2141-V11 in a phase I clinical study using a $3+3$ design in patients with solid tumors with injectable cutaneous, subcutaneous, or nodal lesions.

Results We have completed the first two cohorts without DLTs. In $5 / 6$ patients with breast cancer the best overall response was stable disease. In one patient with melanoma intransit disease we saw a complete response in the second dose group. The third dose group is currently enrolling and updated results will be reported at the meeting.

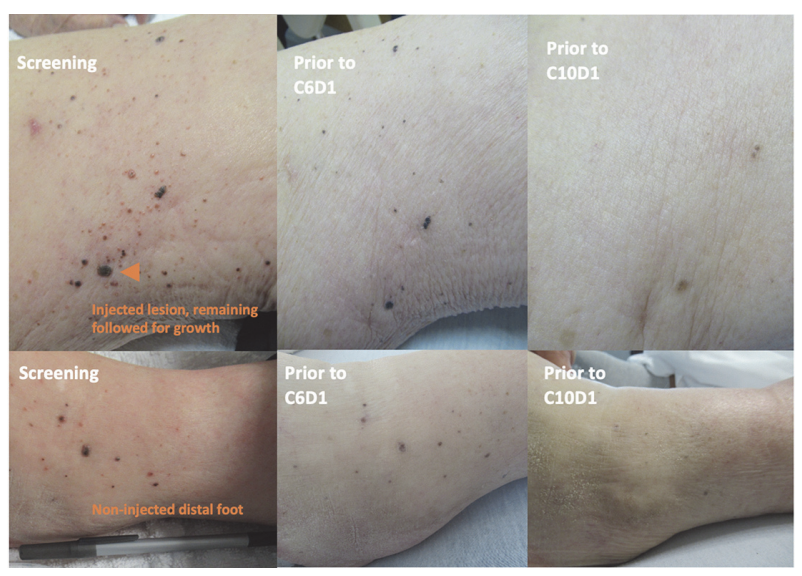

Abstract 496 Figure 1 Intratumoral 2141-V11 leads to both local and distant anti-tumor responses (melanoma)

Conclusions Intratumoral therapy with the Fc-enhanced CD40 agonist 2141-V11 has been demonstrated to be safe, with promising signs of early activity in both injected and distant non-injected lesions (figure 1).

Trial Registration NCT04059588

\section{REFERENCES}

1. Dahan R, Barnhart BC, Li F, Yamniuk AP, Korman AJ, Ravetch JV. Therapeutic Activity of Agonistic, Human Anti-CD40 Monoclonal Antibodies Requires Selective FcyR Engagement. Cancer Cell 2016;0(0):3755-66.

2. Knorr DA, Dahan R, Ravetch JV. Toxicity of an Fc-engineered anti-CD40 antibody is abrogated by intratumoral injection and results in durable antitumor immunity. Proceedings of the National Academy of Sciences 2018. Oct 23;115(43):1104811053.

Ethics Approval This study was approved by the Rockefeller University IRB.

http://dx.doi.org/10.1136/jitc-2021-SITC2021.496 